\title{
Characterization of the SF Agent, an Ehrlichia sp. Isolated from the Fluke Stellantchasmus falcatus, by 16S rRNA Base Sequence, Serological, and Morphological Analyses

\author{
BOHAI WEN, ${ }^{1}$ YASUKO RIKIHISA, ${ }^{1 *}$ SHEIGO YAMAMOTO ${ }^{2}$ NORIHIKO KAWABATA, ${ }^{2}$
} AND PAUL A. FUERST ${ }^{3}$ \\ Department of Veterinary Biosciences ${ }^{1}$ and Department of Molecular Genetics, ${ }^{3}$ The Ohio State University, Columbus, Ohio 43210, and Miyazaki Prefectural Public Health Laboratory, Miyazaki, Japan ${ }^{2}$
}

\begin{abstract}
The organism designated the SF agent was originally isolated in Japan in 1962 from Stellantchasmus falcatus metacercaria parasitic on gray mullet fish. The SF agent resembles members of the genus Ehrlichia morphologically and exhibits weak antigenic cross-reactivity with Ehrlichia sennetsu. This organism causes mild clinical signs in dogs, but severe splenomegaly and lymphadenopathy in mice. This suggests that the SF agent may be similar to either Neorickettsia helminthoeca, an intracellular parasite of a fluke and the cause of salmon poisoning disease in dogs, or $E$. sennetsu, the causative agent of human sennetsu ehrlichiosis in Japan and Malaysia. In order to determine the phylogenetic relationship between the SF agent and other ehrlichial species, the 16S rRNA gene was amplified by the PCR and sequenced. The SF agent sequence was most closely related to the sequences of Ehrlichia risticii (level of sequence similarity, 99.1\%), the causative agent of Potomac horse fever, and $E$. sennetsu (level of sequence similarity, $98.7 \%$ ). The next most similar sequence was that of $N$. helminthoeca, but the level of sequence similarity was only $93.7 \%$. E. sennetsu, E. risticii, the SF agent, and $N$. helminthoeca formed a distinct cluster that was separated from all other ehrlichial species. As determined by immunofluorescence labeling, antiserum against the SF agent cross-reacted strongly with $E$. sennetsu, $E$. risticii, and $N$. helminthoeca. When three genetically distinct ehrlichial isolates obtained from horses with Potomac horse fever were compared with the SF agent, we found that the SF agent was most closely related to Ohio isolate 081 , followed by Illinois ${ }^{T}(T=$ type strain) and a Kentucky isolate. We observed strong antigenic cross-reactivities and similarities in Western blot (immunoblot) reaction profiles when we compared the SF agent, $E$. risticii, and $E$. sennetsu; however, weaker antigenic cross-reactivity was observed when the SF agent and $N$. helminthoeca were compared. Our results indicate that the SF agent is antigenically more closely related to $E$. risticii and $E$. sennetsu than to $N$. helminthoeca. The biological and antigenic characteristics and the $16 \mathrm{~S}$ rRNA sequence data suggest that the SF agent is a new species that belongs to the genus Ehrlichia.
\end{abstract}

Ehrlichiae are small, gram-negative, obligately intracellular bacteria that are the etiological agents of ehrlichiosis in dogs, horses, ruminants, and humans $(11,14)$. Recently, many new Ehrlichia species have been isolated and/or identified in the United States and Japan $(1,2,4,16,17)$. Biological, immunological, and molecular biological studies of these organisms have significantly increased our understanding of ehrlichiae.

An organism designated the SF agent was first isolated by Fukuda et al. (7) in Japan in 1962 from Stellantchasmus falcatus metacercaria parasitic on gray mullet fish. On the basis of the results of morphological, biological, and serological studies, this organism has been recognized as a member of the tribe Ehrlichieae (7-9, 11). The SF agent exhibits weak antigenic cross-reactivity with Ehrlichia sennetsu (7), the causative agent of human sennetsu ehrlichosis in Japan and Malaysia. Like $E$. sennetsu, the SF agent causes severe splenomegaly and lymphadenopathy in mice (7), but it does not kill infected mice, unlike E. sennetsu (7). The SF agent causes mild clinical signs (fever) in dogs and can be reisolated from the blood of infected dogs (9), like Neorickettsia helminthoeca, a rickettsial parasite of the fluke Nanophyetus salmincola and the cause of salmon poisoning disease in dogs. These observations suggest that the

\footnotetext{
* Corresponding author. Mailing address: Department of Veterinary Biosciences, College of Veterinary Medicine, 1925 Coffey Rd., Columbus, OH 43210-1093. Phone: (614) 292-9677. Fax: (614) 2926473.
}

SF agent may be similar to Neorickettsia helminthoeca or E. sennetsu.

In this study, in order to determine the phylogenetic relationship between the SF agent and other ehrlichial species, the SF agent 16S rRNA gene was amplified by the PCR and sequenced. In this paper the results of a comparison of the $16 \mathrm{~S}$ rRNA gene sequences and Western blot (immunoblot) patterns of the SF agent and previously described ehrlichial species and morphological characteristics of the SF agent are described. On the basis of our results, we suggest that the SF agent is a new Ehrlichia species that is most closely related to Ehrlichia risticii, the causative agent of Potomac horse fever, and $E$. sennetsu and that the next most closely related organism is Neorickettsia helminthoeca.

\section{MATERIALS AND METHODS}

Ehrlichia spp. and cell culture. The spleen of a ddY mouse that had been infected with the Hirose strain (a 1967 isolate) of the SF agent in $10 \mathrm{ml}$ of minimum essential medium (GIBCO, Grand Island, N.Y.) was homogenized by using a Cellector tissue sieve (VWR Scientific, Piscataway N.J.), and the resulting homogenate was centrifuged at $300 \times g$ for $3 \mathrm{~min}$. The pellet was resuspended in $5 \mathrm{ml}$ of minimum essential medium containing $10 \%$ fetal bovine serum (Atlanta Biologicals, Norcross, Ga.) and $2 \mathrm{mM}$ L-glutamine (GIBCO), and the resulting suspension was sonicated at a power output setting of 4 for $20 \mathrm{~s}$ with a model W-380 ultrasonic processor (Heat Systems Ultrasonics, Farmingdale, N.Y.). The sonicated suspension was centrifuged at $300 \times g$ for $3 \mathrm{~min}$, and $3 \mathrm{ml}$ of the supernatant was overlaid on a semiconfluent dog macrophage cell line DH82 (11) monolayer in a $25-\mathrm{cm}^{2}$ culture flask. The cells were incubated at $37^{\circ} \mathrm{C}$ in $5 \% \mathrm{CO}_{2}$-air. Erhlichia canis and Neorickettsia helminthoeca were also cultured in DH82 cells. E. sennetsu Miyayama, E. risticii Illinois $^{\mathrm{T}}(\mathrm{T}=$ type strain), and isolates obtained from horses with clinical signs of Potomac horse fever, includ- 


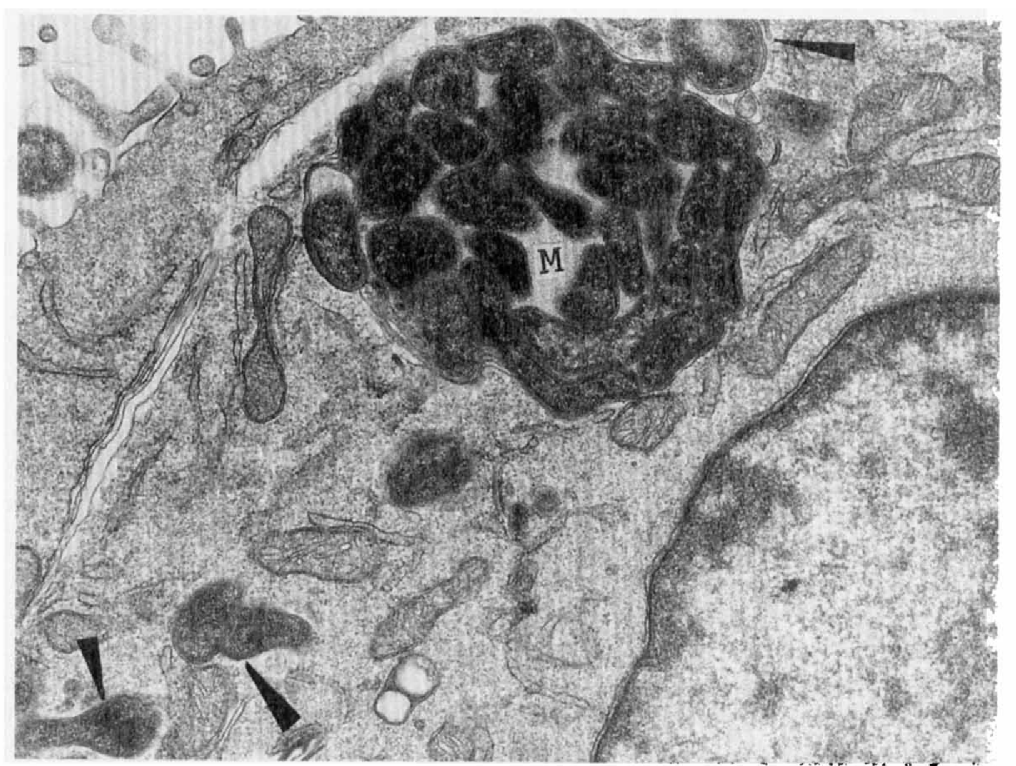
FIG. 1. Ultrastructure of the SF agent in DH82 cells. Note the multiple organisms in an inclusion (a morula [M]) and several individual organisms (arrowheads)
in the cytoplasm. Magnification, $\times 17,400$.

ing isolate 081 and a Kentucky isolate (strain $\mathrm{OV}$ ), were cultured in $\mathrm{P}_{388 \mathrm{D}_{1} \text { cells }}$ by using RPMI 1640 medium (GIBCO) (3).

Ehrlichial agents were purified from the infected host cells by using Sephacryl S-1000 column chromatography as described previously (13).

Preparation of mouse anti-SF agent serum. Nine CF1 mice (Harlan Sprague Dawley, Indianapolis, Ind.) were inoculated intraperitoneally with SF agentinfected DH82 cells $\left(50 \%\right.$ of cells infected; $10^{6}$ cells suspended in $0.1 \mathrm{ml}$ of minimum essential medium per mouse). After 3 weeks, another identical dose of infected cells was inoculated intraperitoneally into the mice to boost their immune responses to the SF agent. Blood was obtained from the inoculated mice 3 weeks after the second inoculation, and the sera from the nine mice were pooled.

Western blot analysis. Western immunoblotting was performed as described previously (12). The purified ehrlichial antigens ( $40 \mu \mathrm{g}$ per lane) were separated by sodium dodecyl sulfate-polyacrylamide gel electrophoresis in a $12 \%$ polyacrylamide slab gel. The separated antigens in the gel were transferred to a nitrocel- lulose membrane with an electrotransblotter (Enprotech, Hyde Park, Mass.) at a constant current of $100 \mathrm{~mA}$ for $45 \mathrm{~min}$; the membrane was blocked in $5 \%$ milk in phosphate-buffered saline and then incubated at $4{ }^{\circ} \mathrm{C}$ overnight with mouse anti-SF agent serum (the homologous titer was 1:10,240; before incubation, the serum was absorbed with lysates containing $10^{7} \mathrm{DH} 82$ cells and $10^{7} \mathrm{P} 388 \mathrm{D}_{1}$ cells per $\mathrm{ml}$ of serum at room temperature for $2 \mathrm{~h}$ ), anti-E. risticii horse serum (titer, 1:1,280), anti-E. sennetsu horse serum (titer, 1:640), and anti-Neorickettsia helminthoeca dog NH4 serum (titer, 1:1,280) diluted 1:20. After three rinses, the membrane was incubated at room temperature for $2 \mathrm{~h}$ with alkaline phosphatase-conjugated affinity-purified anti-mouse, anti-horse, or anti-dog immunoglobulin G (Kirkegaard and Perry Laboratories, Inc., Gaithersburg, Md.) diluted 1:500

Immunofluorescent-antibody test. The host cells infected with ehrlichial species were placed onto 12-well Teflon-coated glass slides (Cel-Line Associates, Inc., Newfield, N.J.) at a concentration of $10^{3}$ cells per well. After the preparations were dried at room temperature, the cells were fixed with cold acetone. The

TABLE 1. Matrix showing corrected nucleotide distances and nucleotide similarity

\begin{tabular}{|c|c|c|c|c|c|c|c|c|c|c|c|c|}
\hline \multirow[b]{2}{*}{ Organism } & \multicolumn{12}{|c|}{ Corrected nucleotide distance or $\%$ nucleotide similarity } \\
\hline & 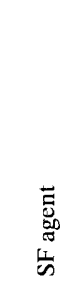 & 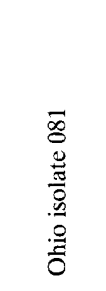 & 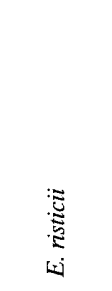 & 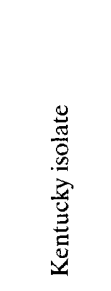 & 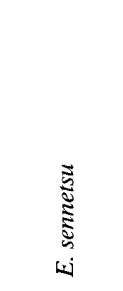 & 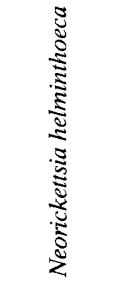 & 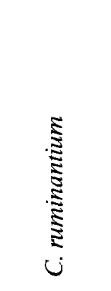 & 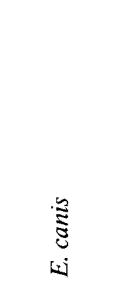 & 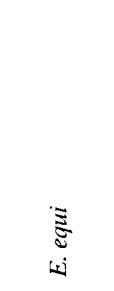 & 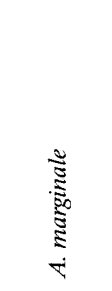 & 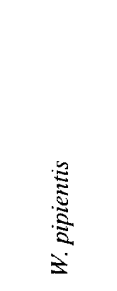 & 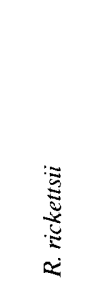 \\
\hline SF agent & & 0.0078 & 0.0098 & 0.0106 & 0.0106 & 0.0473 & 0.1667 & 0.1700 & 0.1661 & 0.1680 & 0.1737 & 0.1885 \\
\hline Ohio isolate 081 & 99.22 & & 0.0036 & 0.0036 & 0.0029 & 0.0442 & 0.1673 & 0.1702 & 0.1679 & 0.1684 & 0.1767 & 0.1862 \\
\hline E. risticii & 99.02 & 99.64 & & 0.0035 & 0.0078 & 0.0442 & 0.1669 & 0.1739 & 0.1653 & 0.1654 & 0.1769 & 0.1885 \\
\hline Kentucky isolate & 98.94 & 99.64 & 99.65 & & 0.0022 & 0.0439 & 0.1669 & 0.1717 & 0.1657 & 0.1671 & 0.1747 & 0.1886 \\
\hline E. sennetsu & 98.95 & 99.72 & 99.23 & 99.78 & & 0.0467 & 0.1655 & 0.1692 & 0.1634 & 0.1659 & 0.1719 & 0.1865 \\
\hline Neorickettsia helminthoeca & 95.44 & 95.73 & 95.73 & 95.76 & 95.50 & & 0.1618 & 0.1619 & 0.1628 & 0.1690 & 0.1740 & 0.1829 \\
\hline C. ruminantium & 85.29 & 85.27 & 85.29 & 85.29 & 85.39 & 85.65 & & 0.0271 & 0.0761 & 0.0773 & 0.1299 & 0.1742 \\
\hline E. canis & 85.03 & 85.03 & 84.75 & 84.91 & 85.10 & 85.62 & 97.34 & & 0.0786 & 0.0803 & 0.1367 & 0.1844 \\
\hline E. equi & 85.32 & 85.19 & 85.39 & 85.36 & 85.53 & 85.57 & 92.85 & 92.64 & & 0.0361 & 0.1339 & 0.1791 \\
\hline A. marginale & 85.19 & 85.17 & 85.40 & 85.27 & 85.36 & 85.12 & 92.76 & 92.48 & 96.49 & & 0.1423 & 0.1793 \\
\hline$W$. pipientis & 84.80 & 84.57 & 84.57 & 85.72 & 85.94 & 84.73 & 88.32 & 87.76 & 87.95 & 87.31 & & 0.1797 \\
\hline R. rickettsii & 83.52 & 83.69 & 83.52 & 83.51 & 83.68 & 83.93 & 84.64 & 83.88 & 84.27 & 84.27 & 84.26 & \\
\hline
\end{tabular}

"The values on the upper right are Kimura two-parameter corrected nucleotide distances, and the values on the lower left are percent nucleotide similarity. A total of 1,464 nucleotides were used in the analysis. 

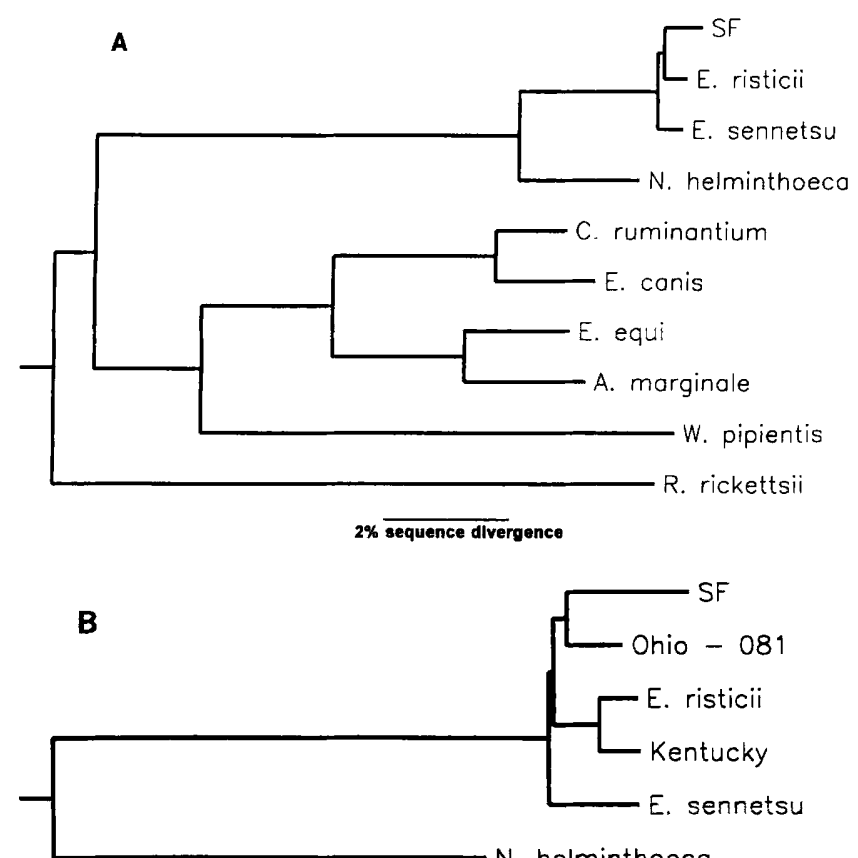

$0.5 \% \overline{\text { sequence divergence }}$

FIG. 2. Phylogenetic relationships of the SF agent and other members of the tribe Ehrlichieae. (A) Phylogenetic relationships of the SF agent, Neorickettsia helminthoeca, E. sennetsu, and E. risticii. (B) Phylogenetic relationships of the SF agent, Ohio isolate 081 , and the Kentucky isolate.

cells were incubated with serially diluted mouse anti-SF agent serum at $37^{\circ} \mathrm{C}$ for $1 \mathrm{~h}$ and then incubated with fluorescein isothiocyanate-conjugated goat antimouse immunoglobulin G (Organon Teknika Co., West Chester, Pa.) at a dilution of 1:100 at $37^{\circ} \mathrm{C}$ for $1 \mathrm{~h}$. The slides were observed with an epifluorescence microscope (Nikon, Inc., Garden City, N.Y.).

Electron microscopy. SF agent-infected DH82 cells were fixed overnight at $44^{\circ} \mathrm{C}$ in a mixture containing $5 \%$ glutaraldehyde, $2.5 \%$ paraformaldehyde, and $0.03 \%$ trinitrophenol in $0.1 \mathrm{M}$ sodium cacodylate buffer $(\mathrm{pH} 7.4)$. The cells were washed twice with $0.1 \mathrm{M}$ sodium cacodylate buffer, postfixed with $1 \%$ osmium tetroxide in $1.5 \%$ potassium ferrocyanide for $1 \mathrm{~h}$, block stained with $1 \%$ uranyl acetate, dehydrated in a graded ethanol-propylene oxide series, and embedded in Poly/ Bed 812 resin (Polysciences, Inc., Warrington, $\mathrm{Pa}$.). Uttrathin sections were stained with uranyl acetate and lead citrate and examined with a Philips model 300 electron microscope at $60 \mathrm{kV}$.

Extraction of DNA. The DNA of chromatography-purified SF agent was extracted with a QIAamp tissue kit (QIAGEN, Inc., Chatsworth, Calif.) by using the protocol recommended by the manufacturer. This DNA was used as the template in the PCR.

Amplification of the 16S rRNA gene of the SF agent. The 16S rRNA gene was amplified with $5^{\prime}$-end primer A-17 (5'-GTTTGATCATGGCTCAG-3') and $3^{\prime}$. end primer 3-17 (5'-AAGGAGGTAATCCAGCC-3'), which are universal primers for the $16 \mathrm{~S}$ rRNA gene sequences of prokaryotic cells. The PCR was performed as described previously (17). The amplified DNA fragment that was the expected size (approximately $1.5 \mathrm{~kb}$ ) was purified from a $1 \%$ low-melting-temperature agarose gel (GIBCO BRL) by using a PCR DNA purification system (Promega, Madison, Wis.). This DNA was used as the template for cycle sequencing.

DNA sequencing of the 16S rRNA PCR product. The sequences of both DNA strands were determined by using the dideoxynucleotide method, a doublestranded DNA cycle sequencing system (GIBCO BRL), and the protocol recommended by the manufacturer. The forward and reverse sequencing primers used were derived from the conserved sequences of known ehrlichial 16S rRNA genes on the basis of the results of an analysis in which we used the program OLIGO (National Biosciences, Inc., Hamel, Mich.). The sequencing primers used were primers A-17, 3-17, ER323-341 (5'-CTACGGGAGGCAGCAG CAGTG-3), ER757-775 (5'-TAGTCCACGCTGTAAACG-3'), ER1043-1062 (5'-TAAGTCCCGCAACGAGC-3'), ER864-846 (5'-GAGTTTTAGTCTTGC GACC-3'), ER493-476 (5'-GTATTACCGCGGCGCTG-3'), and ER247-228 (5'-CATTACCCCACCAACTAGC-3'). The primer positions were designated on the basis of the $16 \mathrm{~S}$ rRNA sequence of $E$. risticii Illinois $^{\mathrm{T}}$. A $5 \%$ Long Ranger gel (AT Biochem, Malvern, Pa.) was used for sequencing in order to determine more than 400 bases with each primer.

Computer analyses of DNA sequences. The corrected levels of nucleotide divergence of $16 \mathrm{~S}$ rRNA genes were calculated by using DNADIST from PHYLIP version 3.1 (5) with the Kimura two-parameter correction. A phylogenetic tree for the taxa studied was constructed by the neighbor-joining method (NEIGHBOR in PHYLIP) (5) on the basis of distance matrix data. Only homologous sites at which the 16S rRNA gene sequences were aligned unambiguously were included in the phylogenetic analysis.

Nucleotide sequence accession numbers. The 16S rRNA nucleotide sequences used in this study were obtained from the GenBank database. The GenBank nucleotide sequence accession numbers for the organisms which we studied are as follows: Anaplasma marginale, M60313; Cowdria ruminantium, X62432; E. canis, M73221; Ehrlichia equi, M73223; E. sennetsu, M73225; E. risticii, M21290; Neorickettsia helminthoeca, U12457; Rickettsia rickettsii, U11021; Wolbachia pipientis, X61768; and SF agent, U34280.

\section{RESULTS}

Electron microscopy of the SF agent. The SF agent was originally maintained by passage in mice (6). Although it had been cultured in RK-13 (rabbit kidney epithelial) cells once (7), the SF agent could no longer be cultured at the beginning of our study. In this study, the SF agent was effectively cultured and maintained in a continuous dog cell line for the first time. The ultrastructure of the SF agent in the cytoplasm of DH82 cells is shown Fig. 1. Ultrastructurally, the SF agent resembles other ehrlichial species (11).

Analysis of the 16S rRNA sequence. A single DNA fragment $(1.5 \mathrm{~kb})$ was amplified by PCR from the genomic DNA of the SF agent with a pair of primers that flanked the $16 \mathrm{~S}$ rRNA gene. This DNA fragment was sequenced, and a 1,477-base sequence was obtained. The sequence of the SF agent was aligned with the 16S rRNA gene sequences of other members of the tribe Ehrlichieae and with the sequences of closely related intracellular bacteria. The highest levels of similarity observed were the levels of similarity between the SF agent sequence and the sequences of $E$. risticii Illinois ${ }^{T}$ (level of

TABLE 2. Major antigenic polypeptides detected by Western blot analysis and immunofluorescent-antibody titers with mouse anti-SF agent serum

\begin{tabular}{|c|c|c|c|c|c|c|}
\hline \multirow[b]{2}{*}{$\begin{array}{l}\text { Size of polypeptide } \\
(\mathrm{kDa})\end{array}$} & \multicolumn{6}{|c|}{ Presence with the following antigens": } \\
\hline & 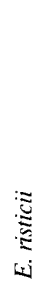 & 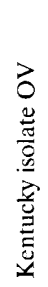 & 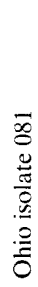 & 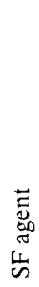 & 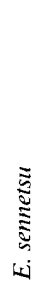 & 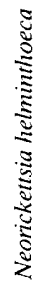 \\
\hline 64 & + & & & + & & \\
\hline 62 & & & & & + & + \\
\hline 58 & & + & + & + & + & \\
\hline 54 & & & & & + & \\
\hline 51 & + & & & & & \\
\hline 50 & + & + & + & + & & \\
\hline 44 & + & + & + & & + & \\
\hline 43 & & & + & & + & \\
\hline 42 & & & + & + & & \\
\hline 41 & & & & + & + & + \\
\hline 34 & & & + & & & \\
\hline 32 & & & & + & & \\
\hline 28 & & + & + & + & + & \\
\hline 26 & & & & + & + & \\
\hline
\end{tabular}

a No polypeptides were detected with antigens to various Ehrlichia spp. The immunofluorescent-antibody titer of anti-SF agent serum was 1:10,240. 


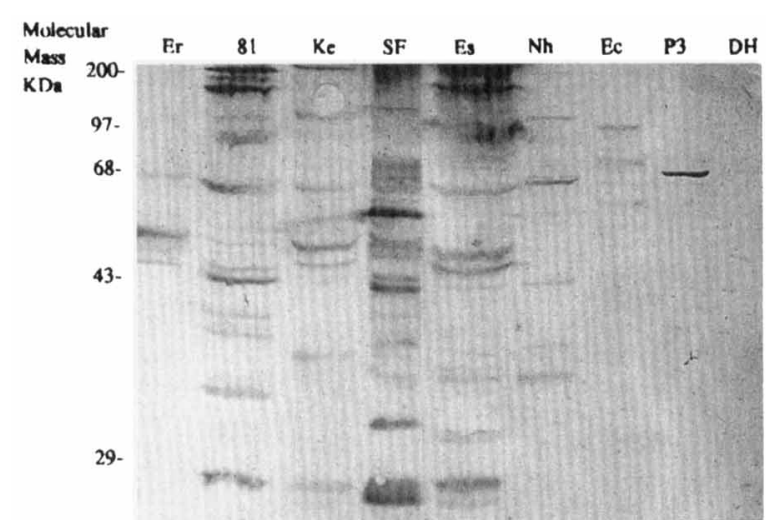

a. Mouse anti-SF agent serum

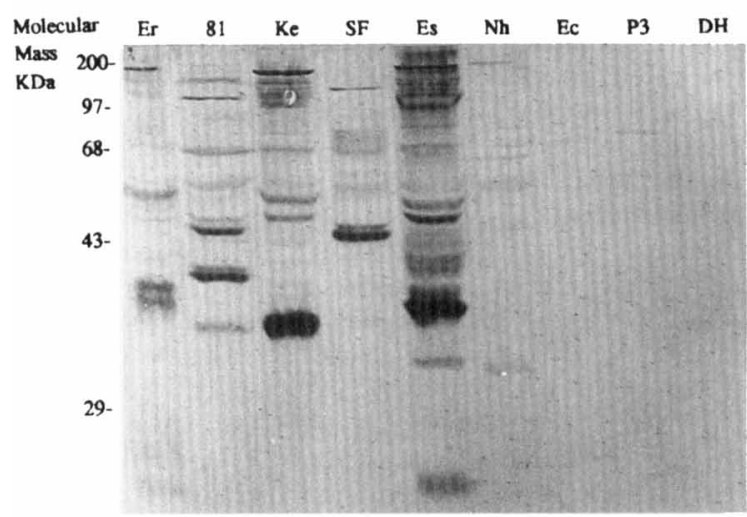

c. Pony 41 anti-E. sennetsu serum

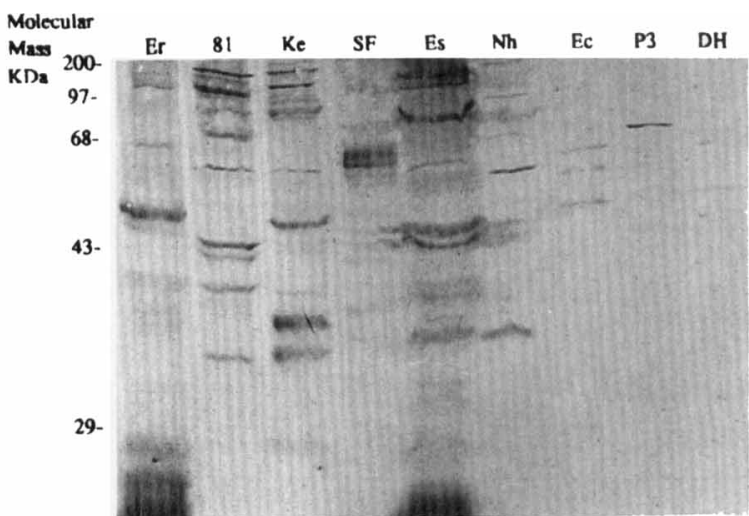

b. Pony 19 anti-E. risticii serum

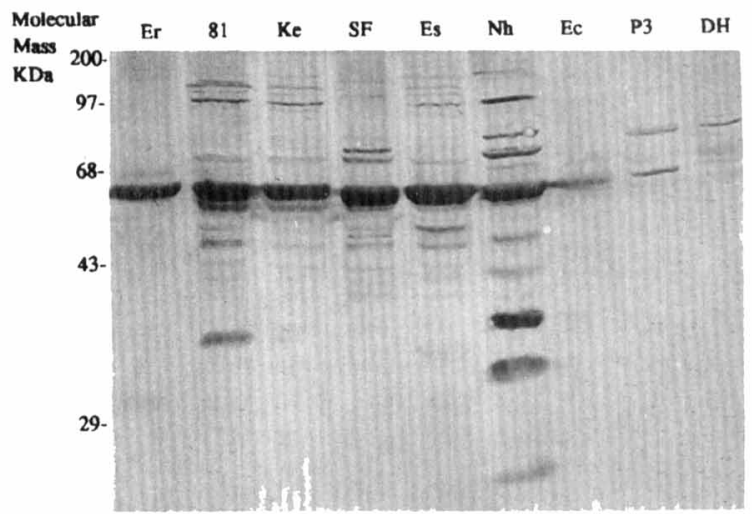

d. Dog anti-N. helminthoeca serum

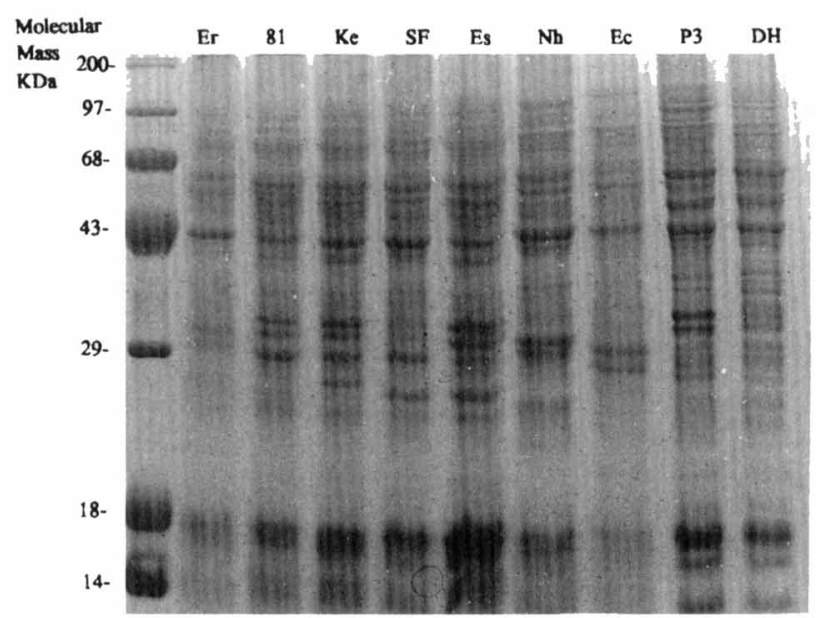

e. SDS-PAGE stained with Coomassie blue

FIG. 3. Protein profiles and immunoblot analysis of antigens stained with Coomassie blue or four different antisera. The negative controls used were DH82 cells (lanes DH) and P388D, cells (lanes P3). Lanes Ec, E. canis; lanes Nh, Neorickettsia helminthoeca; lanes Es, E. sennetsu; lanes SF, SF agent; lanes Ke, Kentucky isolate OV; lanes 81, Ohio isolate 081; lanes Er, E. risticii. SDS-PAGE, sodium dodecyl sulfate-polyacrylamide gel electrophoresis.

similarity, 99.1\%) and E. sennetsu (level of similarity, 98.7\%). The next most similar sequence was that of Neorickettsia helminthoeca (10) (level of similarity, 93.7\%). In our subsequent phylogenetic analysis, we used the sequences which could be unambiguously aligned for all species. The levels of sequence similarity and corrected levels of nucleotide divergence used for the phylogenetic comparisons are shown in Table 1 . The phylogenetic tree constructed on the basis of these data is shown in Fig. 2A. Our data showed that the SF agent, $E$. risticii, E. sennetsu, and Neorickettsia helminthoeca joined together to form a distinct cluster that was separated from all other species. To examine the relationships further, we included in the 
phylogenetic analysis two additional 16S rRNA gene sequences, the sequence of Ohio isolate 081 and the sequence of a Kentucky isolate obtained from horses that had clinical signs of Potomac horse fever (16). The sequences that were most closely related to the SF agent sequence were the sequences of isolate 081 (level of similarity, 99.2\%), E. risticii Illinois $^{\mathrm{T}}$ (level of similarity, $99.1 \%$ ), and the Kentucky isolate (level of similarity, 99.0\%) (Fig. 2B).

Immunofluorescent-antibody test and Western blot analysis. The serum obtained from SF agent-inoculated mice was used to study antigenic cross-reactivity with related ehrlichial species by an immunofluorescent-antibody test. Cross-reactivity was observed with isolate 081 , the $E$. risticii type strain, the Kentucky isolate, E. sennetsu, and Neorickettsia helminthoeca (Table 2). Only weak reactivity was observed with $E$. canis (Table 2).

When we used the mouse anti-SF agent serum as the probe, more than 10 polypeptide antigens in the SF agent were recognized in the Western immunoblot analysis (Fig. 3a). E. sennetsu, Ohio isolate 081 , and the Kentucky isolate had more antigens in common with the SF agent than the E. risticii type strain, Neorickettsia helminthoeca, or E. canis did. The major polypeptide antigens of ehrlichial species and Neorickettsia helminthoeca that reacted with the anti-SF agent serum are shown in Table 2.

When we used anti-E. risticii serum, many polypeptide antigens (molecular sizes, 30 to $200 \mathrm{kDa}$ ) were detected in all of the organisms except $E$. canis (Fig. 3b). The reactivity of pony 19 serum was very similar to the reactivity of anti-SF agent serum. A $43-\mathrm{kDa}$ polypeptide antigen was found in the SF agent and Ohio isolate 081 . A 46-kDa antigen was commonly identified in the SF agent, isolate 081, and E. sennetsu. In addition, a 48-kDa antigen was identified in the SF agent, Ohio isolate 081, E. risticii, and Neorickettsia helminthoeca (Fig. 3b).

Strong cross-reacting profiles were observed with all of the organisms except Neorickettsia helminthoeca and E. canis when we used horse anti-E. sennetsu serum (Fig. 3c).

A 64-kDa polypeptide antigen from all of the agents except E. canis reacted strongly with dog anti-Neorickettsia helminthoeca serum (Fig. 3d), as reported previously (12). Only one weak 64-kDa antigen was observed in E. canis (Fig. 3d).

Uninfected P388D 1 and DH82 host cells were used as the negative controls in the Western blot analysis. When anti-SF agent, anti-E. risticii, anti-E. sennetsu, and anti-Neorickettsia heliminthoeca sera were used, only a few polypeptides exhibited weak cross-reactivity in the uninfected host cells (Fig. 3).

\section{DISCUSSION}

The SF agent was found during a systematic search for the etiologic agent of sennetsu ehrlichiosis in Miyazaki Prefecture, the area where sennetsu ehrlichiosis is endemic in Japan, in the 1960 s. On the basis of the results of epidemiologic studies, researchers suspected that sennetsu ehrlichiosis patients may have contracted the disease by eating raw fish infested with flukes harboring $E$. sennetsu (9). By feeding 96 people raw gray mullet fish caught in the river in the area where sennetsu ehrlichiosis is endemic, the researchers reproduced sennetsu ehrlichiosis-like disease in five individuals (6). Japanese scientists then isolated the SF agent from the fluke that parasitized gray mullet fish. However, this agent appeared to be distinct from E. sennetsu that was isolated from human patients on the basis of the clinical and pathologic responses of mice inoculated with this agent and the results of serological reactions (7-9). While the results of electron microscopy, biological characteristics, and antigenic cross-reactivity data strongly sug- gested that the SF agent belongs to an ehrlichial species (11), the phylogenetic relationships with other members of the tribe Ehlichieae were unknown.

In this study, we found that the 16S rRNA gene of the SF agent was most similar to the $16 \mathrm{~S}$ rRNA genes of E. risticii and $E$. sennetsu. The levels of sequence divergence between the SF agent sequence and the sequences of $E$. risticii and $E$. sennetsu were 0.9 and $1.3 \%$, respectively. The $16 \mathrm{~S}$ rRNA gene of bacteria is highly conserved (18), and the levels of $16 \mathrm{~S}$ rRNA gene divergence have often been found to be low in related intracellular bacteria. For instance, Anderson et al. (1) found that the levels of divergence between species belonging to the genus Ehrlichia ranged from 0.1 to $16 \%$ in an analysis of $16 \mathrm{~S}$ rRNA gene sequences; however, for strains belonging to the same ehrlichial species only one to three bases were different (1). Therefore, the SF agent may be a new member of the genus Ehrlichia. On the basis of the results of a comparison of $16 \mathrm{~S}$ rRNA nucleotide sequences, the SF agent is most closely related to $E$. risticii and $E$. sennetsu, while Neorickettsia helminthoeca is the next most closely related species. These phylogentic analysis results were consistent with the results of our immunofluorescent-antibody and immunoblot analyses performed with immune sera against different ehrlichial species. The results of previous serological and biological studies also suggested that the SF agent was closely related to E. sennetsu and Neorickettsia helminthoeca (7-9). Strain 081 and the Kentucky ehrlichial strain, which were isolated from horses with signs of Potomac horse fever, are two strains that were differentiated from the type strain of E. risticii (strain Illinois) on the basis of serological, Western blot, and 16S rRNA gene sequence analysis results $(3,16)$. The SF agent was found to be more closely related to Ohio isolate 081 (level of similarity, $99.2 \%$ ) than to the Kentucky isolate or the type strain of $E$. risticii in the $16 \mathrm{~S}$ rRNA sequence analysis.

Both the SF agent and Neorickettsia helminthoeca were isolated from flukes (11). Since the SF agent, E. risticii, E. sennetsu, and Neorickettsia helminthoeca form a cluster within the tribe Ehrlichieae that is distinct from the clusters formed by the tick-borne ehrlichial species, such as E. canis and Ehrlichia chaffeensis or E. equi and Ehrlichia phagocytophila, our results further support the hypothesis that E. sennetsu and E. risticii may also be transmitted by flukes.

The SF agent can cause severe splenomegaly and lymphadenopathy in mice (7) and mild clinical signs in dogs (9). Whether it infects horses (like E. risticii) or humans and whether it causes disease (like E. sennetsu) remain to be determined.

\section{ACKNOWLEDGMENTS}

We thank Wiwat Chaichanasiriwithaya and Yilan Zhang for providing antigens of some Ehrlichia spp.

This work was supported in part by the Ohio State University equine research fund.

\section{REFERENCES}

1. Anderson, B. E., J. E. Dawson, D. C. Jones, and K. H. Wilson. 1991. Ehrlichia chaffeensis, a new species associated with human ehrlichiosis. J. Clin. Microbiol. 29:2838-2842

2. Anderson, B. E., C. E. Greene, D. C. Jones, and J. E. Dawson. 1992. Ehrlichia ewingii sp. nov., the etiologic agent of canine granulocytic ehrlichiosis. Int. J. Syst. Bacteriol. 42:299-302.

3. Chaichanasiriwithaya, W., Y. Rikihisa, S. Yamamoto, S. M. Reed, T. B. Crawford, L. E. Perryman, and G. H. Palmer. 1994. Antigenic, morphologic, and molecular characterization of new Ehrlichia risticii isolates. J. Clin. Microbiol. 38:3026-3033.

4. Chen, S. M., J. S. Dumler, J. S. Bakken, and D. H. Walker. 1994. Identification of a granulocytotropic Ehrlichia species as the etiologic agent of human disease. J. Clin. Microbiol. 32:589-595. 
5. Felsenstein, J. 1989. PHYLIP—phylogeny inference package (version 3.3). Cladistics 5:164-166.

6. Fukuda, T., T. Sasahara, and T. Kitao. 1972. Studies on the causative agent of "Hyuganetsu" disease. X. Vector. J. Jpn. Assoc. Infect. Dis. 36:235-241. (In Japanese.)

7. Fukuda, T., T. Sasahara, and T. Kitao. 1973. Studies on the causative agent of "Hyuganetsu" disease. XI. Characteristics of rickettsia-like organism isolated from metacercaria of Stellantchasmus falcatus parasitic in grey mullet. J. Jpn. Assoc. Infect. Dis. 47:474-482. (In Japanese.)

8. Fukuda, T., and S. Yamamoto. 1979. Antigenic analysis and ultrastructure of the rickettsia-like organism isolated from Stellantchasmus falcatus. J. Jpn. Assoc. Infect. Dis. 53:713-716. (In Japanese.)

9. Fukuda, T., and S. Yamamoto. 1981. Neorickettsia-like organism isolated from metacercaria of a fluke, Stellantchasmus falcatus. Jpn. J. Med. Sci. Biol. 34:103-107.

10. Pretzman, C., D. Ralph, D. R. Stothard, P. A. Fuerst, and Y. Rikihisa. 1995. 16 S rRNA gene sequence of Neorickettsia helminthoeca: phylogenetic alignment with members of the genus Ehrlichia. Int. J. Syst. Bacteriol. 45:207-211.

11. Rikihisa, Y. 1991. The tribe Ehrlichieae and ehrlichial diseases. Clin. Microbiol. Rev. 4:286-308.
12. Rikihisa, Y. 1991. Cross-reacting antigens between Neorickettsia helminthoeca and Ehrlichia species, shown by immunofluorescence and Western immunoblotting. J. Clin. Microbiol. 29:2024-2029.

13. Rikihisa, Y., S. A. Ewing, J. C. Fox, A. G. Siregar, F. H. Pasaribu, and M. B. Malole. 1992. Analysis of Ehrlichia canis and a canine granulocyte Ehrlichia infection. J. Clin. Microbiol. 30:143-148.

14. Ristic, M., and D. Huxoll. 1984. Tribe II. Ehrlichieae, p. 704-711. In N. R. Krieg and J. G. Holt (ed.), Bergey's manual of systematic bacteriology, vol. 1. The Williams \& Wilkins Co., Baltimore.

15. Weisburg, W. G., M. E. Dobson, J. E. Samuel, G. A. Dasch, L. Mallavia, O. Baca, L. Mandelco, J. E. Sechrest, E. Weiss, and C. R. Woese. 1989. Phylogenetic diversity of the rickettsiae. J. Bacteriol. 171:4202-4206.

16. Wen, B., Y. Rikihisa, P. A. Fuerst, and W. Chaichanasiriwithaya. 1995. Diversity of 16S rRNA genes of new Ehrlichia strains isolated from horses with clinical signs of Potomac horse fever. Int. J. Syst. Bacteriol. 45:315-318.

17. Wen, B., Y. Rikihisa, J. Mott, P. A. Fuerst, M. Kawahara, and C. Suto. 1995. Ehrlichia muris sp. nov., identified on the basis of $16 \mathrm{~S}$ rRNA base sequences and serological and biological characteristics. Int. J. Syst. Bacteriol. 45:250254.

18. Woese, C. R. 1987. Bacterial evolution. Microbiol. Rev. 51:221-271. 